\title{
Changes in marrow adipose tissue with short-term changes in weight in premenopausal women with anorexia nervosa
} \author{
Clifford J Rosen ${ }^{4}$ and Anne Klibanski, ${ }^{1,2}$ \\ ${ }^{1}$ Neuroendocrine Unit, Massachusetts General Hospital, ${ }^{2}$ Harvard Medical School, ${ }^{3}$ Department of Radiology, \\ Massachusetts General Hospital, Boston, Massachusetts, USA, and ${ }^{4}$ Maine Medical Center Research Institute, \\ Scarborough, Maine, USA
}

Pouneh K Fazelii,2, Alexander T Faje ${ }^{1,2}$, Miriam A Bredella ${ }^{2,3}$, Sai Polineni', Stephen Russell', Megi Resulaj',

Correspondence should be addressed to P K Fazeli Email

pkfazeli@partners.org

\begin{abstract}
Objective: In anorexia nervosa, a psychiatric disease characterized by self-induced starvation and a model of chronic undernutrition, levels of subcutaneous (SAT) and visceral (VAT) adipose tissue are low, whereas marrow adipose tissue (MAT) levels are elevated compared to normal-weight women. The reason for this paradoxical elevation of an adipose tissue depot in starvation is not known. We sought to understand changes in MAT in response to subacute changes in weight and to compare these changes with those of other fat depots and body composition parameters.

Design and methods: We conducted a 12-month longitudinal study including 46 premenopausal women ( $n=26$ with anorexia nervosa and $n=20$ normal-weight controls) with a mean (s.E.M.) age of $28.2 \pm 0.8$ years. We measured MAT, SAT, VAT and bone mineral density (BMD) at baseline and after 12 months.

Results: At baseline, SAT $(P<0.0001)$, VAT $(P<0.02)$ and BMD of the spine and hip $(P \leq 0.0002)$ were significantly lower and vertebral and metaphyseal MAT $(P \leq 0.001)$ significantly higher in anorexia nervosa compared to controls. Weight gain over 12 months was associated with increases not only in SAT and VAT, but also epiphyseal MAT $(P<0.03)$.

Changes in epiphyseal MAT were positively associated with changes in BMD $(P<0.03)$.

Conclusions: In contrast to the steady state, in which MAT levels are higher in anorexia nervosa and MAT and BMD are inversely associated, short-term weight gain is associated with increases in both MAT and BMD. These longitudinal data demonstrate the dynamic nature of this fat depot and provide further evidence of its possible role in mineral metabolism.
\end{abstract}

\section{Introduction}

States of chronic undernutrition are characterized by low subcutaneous and visceral fat stores. Anorexia nervosa, a primary psychiatric disorder predominantly affecting women in which individuals are unable to maintain a normal weight due to self-induced starvation, serves as a model of chronic undernutrition. In addition to lower subcutaneous and visceral fat stores $(1,2)$, women with anorexia nervosa also have significantly lower BMD (3) and a higher rate of fracture as compared to normal-weight controls of similar age $(4,5,6)$. Although hormonal adaptations to undernutrition, including functional hypothalamic amenorrhea and growth hormone resistance, contribute to this low BMD state, the pathophysiology of bone loss in anorexia nervosa is not fully understood (7).

Despite having low levels of subcutaneous and visceral fat, as compared to normal-weight individuals, we have shown that women with anorexia nervosa have higher 
levels of marrow adipose tissue (MAT) (2), an adipose tissue depot residing in the bone marrow microenvironment. The function of MAT is unknown but the fact that levels of MAT are higher in individuals with chronic energy deficits who are actively using other adipose tissue depots as an energy source has led to the hypothesis that MAT may have a significant function. Importantly, data support the concept that MAT may also be a determinant of bone strength and fracture risk. MAT is inversely associated with BMD in multiple populations including women with anorexia nervosa (2), and in adolescents with anorexia nervosa, MAT is associated with decreased estimates of bone strength (8). Similarly, higher levels of vertebral MAT have been associated with decreased bone integrity, as evidenced by vertebral endplate depression and compression fractures (9). Therefore, high levels of MAT may be an important contributor to the decreased bone strength and higher fracture risk characteristic of anorexia nervosa.

We have previously shown that women who have recovered from anorexia nervosa have levels of MAT comparable to normal-weight controls (10) but whether MAT changes with shorter-term changes in weight has not been previously reported. We hypothesized that with short-term weight gain, levels of MAT would decrease toward normal and BMD would increase. We performed a longitudinal, 12-month study in women with anorexia nervosa and normal-weight controls to observe how shortterm changes in weight, body composition and BMD are associated with changes in MAT.

\section{Subjects and methods}

\section{Subjects}

We studied 46 women: 26 with anorexia nervosa (median (interquartile range): $2(6(25,31)$ years $))$ and 20 normalweight controls of comparable age $26.5(24,32)$ years. Women with anorexia nervosa were recruited through referrals from local eating disorder providers and on-line advertisements and control subjects were recruited through on-line advertisements. Subjects with anorexia nervosa met DSM-IV (11) weight and psychiatric criteria. None of the subjects used exogenous estrogen within 3 months of their baseline visit. All normal-weight controls had a normal BMI, a history of regular menstrual cycles and none had a history of an eating disorder. None of the subjects were taking any medications known to affect bone mass, including estrogen or glucocorticoids. Subjects with abnormal thyroid function tests and chronic diseases known to affect BMD (other than anorexia nervosa) were excluded from participation. All subjects with anorexia nervosa were being followed by an outpatient treatment team including a primary care provider and/or therapist at the time of their screening visit. All subjects with anorexia nervosa reported continuing their stable outpatient treatment regimen during the study except for two subjects who were admitted to a residential treatment center for 3 months each. One of these two subjects gained $55.1 \%$ of her baseline weight during the 12-month study and the second subject gained $10.6 \%$ of her baseline weight during the study. The data for the subject who gained $55.1 \%$ of her baseline weight were excluded in a sensitivity analysis examining the association between change in weight and change in MAT (see 'Associations between weight gain and changes in body composition and hormonal parameters' section below).

\section{Study protocol}

All subjects were seen at the Translational and Clinical Research Center at the Massachusetts General Hospital for two study visits, 12 months apart. At the baseline and 12-month study visits, blood was drawn for laboratory studies (fasting), radiologic imaging (described below) was performed, height was measured as the average of three readings on a single stadiometer and subjects were weighed on an electronic scale while wearing a hospital gown.

The study was approved by the Partners Institutional Review Board and complied with the Health Insurance Portability and Accountability Act guidelines. Written informed consent was obtained from all subjects.

\section{Radiologic imaging}

Dual-energy X-ray absorptiometry

All subjects underwent dual-energy X-ray absorptiometry (DXA) to measure areal BMD of the posterior-anterior (PA) lumbar spine (L1-L4), lateral spine (L2-L4), left total hip and left femoral neck using a Hologic Discovery A densitometer (Hologic Inc., Bedford, MA, USA). Coefficients of variation of DXA have been reported as $<2.2 \%$ for bone (12). Baseline BMD data were previously reported for a subset of subjects $(n=21)(13)$. 
Magnetic resonance imaging and ${ }^{1} \mathrm{H}$-magnetic resonance spectroscopy $\left({ }^{1} \mathrm{H}-\mathrm{MRS}\right)$

A single axial magnetic resonance imaging (Siemens Trio, 3T, Siemens Medical Systems) slice through the abdomen at the level of L4 was used to determine abdominal subcutaneous adipose tissue and visceral adipose tissue.

${ }^{1} \mathrm{H}$-magnetic resonance spectroscopy ( ${ }^{1} \mathrm{H}$-MRS) of the L4 vertebral body, the proximal femoral epiphysis, metaphysis and mid-diaphysis was performed to determine lipid content (Siemens Trio, 3T, Siemens Medical Systems). For the L4 vertebra, a voxel measuring $15 \times 15 \times 15 \mathrm{~mm}$ $(3.4 \mathrm{~mL})$ was placed within the $\mathrm{L} 4$ vertebral body. Singlevoxel ${ }^{1} \mathrm{H}$-MRS data were acquired using point-resolved spatially localized spectroscopy (PRESS) pulse sequence without water suppression with the following parameters: TE of $30 \mathrm{~ms}$, TR of $3000 \mathrm{~ms}, 8$ acquisitions, 1024 data points and receiver bandwidth of $2000 \mathrm{~Hz}$. For the femur, a voxel measuring $12 \times 12 \times 12 \mathrm{~mm}^{3}(1.7 \mathrm{~mL})$ was positioned within the proximal femoral epiphysis and single-voxel ${ }^{1} \mathrm{H}$-MRS using the same non-water suppressed PRESS pulse sequence was performed. This process was repeated with voxel placement in the metaphysis at the intertrochanteric region and the mid-diaphysis. Automated procedures for optimization of gradient shimming and transmit and receive gain were used. We have previously reported that the coefficient of variation (CV) for marrow fat quantification in this population, determined by scanning five subjects twice, is $3 \%$ (10).

Fitting of the ${ }^{1} \mathrm{H}$-MRS data was performed using LCModel software (version 6.1-4A) (Stephen Provencher, Oakville, ON, Canada). Data were transferred from the scanner to a Linux workstation and metabolite quantification was performed using eddy current correction and water scaling. A customized fitting algorithm for bone marrow analysis provided estimates for all lipid signals combined $(0.9,1.3$, and $2.3 \mathrm{ppm})$. LCModel bone marrow lipid estimates were automatically scaled to unsuppressed water peak $(4.7 \mathrm{ppm})$ and expressed as lipid to water ratio.

\section{Biochemical assessment}

Leptin was measured by ELISA (R\&D Systems) with an intra-assay CV of $3.2 \%$ and an inter-assay CV of $4.4 \%$. Insulin-like growth factor (IGF)-1 levels were measured by a luminescent immunoassay analyzer (ISYS Analyzer; Immunodiagnostics Corporation, Woburn, MA, USA). The detection limit for IGF- 1 was $4.4 \mathrm{ng} / \mathrm{mL}$, with an intraassay CV of $2.16 \%$ and an inter-assay CV of $5.06 \%$. Peptide YY (PYY) was measured by ELISA (EMD Millipore) with an intra-assay CV of $2.3 \%$ and an inter-assay CV of $7.41 \%$. Adiponectin was measured by ELISA (R\&D Systems) with an intra-assay CV of 3.53\% and an inter-assay CV of 6.5\%. Cortisol was measured by ELISA (ALPCO, Salem, NH, USA) with an intra-assay CV of $5.8 \%$ and an inter-assay $\mathrm{CV}$ of 5.6\%. Insulin and glucose were measured through a clinical laboratory (NorDx Laboratory, Scarborough, ME, USA). Homeostatic model assessment of insulin resistance (HOMA-IR) was calculated using the following equation: (Glucose $(\mathrm{mg} / \mathrm{dL}) \times$ insulin $(\mu \mathrm{U} / \mathrm{mL})) / 405$.

\section{Statistical analysis}

Statistical analysis was performed using JMP Pro 13.0 (SAS Institute) software. If the data were normally distributed, means and standard error of the mean (s.E.M.) are reported and compared using the Student's $t$-test. If the data were not normally distributed, medians and the interquartile range are reported and compared using the Wilcoxon test. Paired sample $t$-tests or Wilcoxon signed-rank test (if data were non-normally distributed) were used to compare changes in body composition parameters between the baseline and 12-month study visits. Pearson correlation coefficients (for normally distributed data) or Spearman's coefficients (for non-normally distributed data) were calculated to assess univariate relationships. A $P$ value of $<0.05$ was considered significant.

\section{Results}

\section{Baseline characteristics of study population}

Baseline characteristics of the study subjects are listed in Table 1. Subjects with anorexia nervosa and normal-weight controls were of similar age $(P=0.99)$. By study design, BMI and \% ideal body weight were significantly lower in women with anorexia nervosa as compared to controls $(P<0.0001$ for both). Women with anorexia nervosa also had significantly less subcutaneous $(P<0.0001)$ and visceral $(P<0.02)$ adipose tissue as compared to normalweight controls.

We measured leptin and IGF-1 levels, as both of these hormones are nutritionally regulated and have been associated with bone metabolism in anorexia nervosa. Treatment with recombinant human leptin, an adipokine primarily secreted by subcutaneous adipocytes, increases levels of markers of bone formation in women with low leptin levels in the setting of hypothalamic amenorrhea (14). Similarly, levels of IGF-1 drop acutely during a 
Table 1 Baseline characteristics of study subjects. We report (mean \pm S.E.M.) or median (interquartile range) when data were not normally distributed.

\begin{tabular}{l}
\hline \\
\hline Age (years) \\
Weight $(\mathrm{kg})$ \\
BMI $\left(\mathrm{kg} / \mathrm{m}^{2}\right)$ \\
\% Ideal body weight \\
Duration of anorexia nervosa (years) \\
Amenorrhea duration (months) \\
Subcutaneous adipose tissue $\left(\mathrm{cm}^{2}\right)$ \\
Visceral adipose tissue $\left(\mathrm{cm}^{2}\right)$ \\
Leptin $(\mathrm{ng} / \mathrm{mL})$ \\
IGF-1 $(\mathrm{ng} / \mathrm{mL})$ \\
PYY $(\mathrm{pg} / \mathrm{mL})$ \\
Adiponectin $(\mu \mathrm{gg} / \mathrm{mL})$ \\
Cortisol $(\mu \mathrm{gg} / \mathrm{dL})$ \\
Glucose $(\mathrm{mg} / \mathrm{dL})$ \\
Insulin $(\mu \mathrm{U} / \mathrm{mL})$ \\
\hline
\end{tabular}

\begin{tabular}{c}
\hline Anorexia nervosa $(n=26)$ \\
\hline $26(25,31)$ \\
$44.5 \pm 1.6$ \\
$16.7 \pm 0.5$ \\
$75.6 \pm 2.0$ \\
$13.0 \pm 1.3$ \\
$46.5(21.8,105.8)$ \\
$57.0 \pm 6.0$ \\
$19.1(10.3,31.2)$ \\
$1.2(0.3,3.2)$ \\
$173 \pm 11$ \\
$105.1(46.1,131.2)$ \\
$11.22(6.40,17.11)$ \\
$15.8(11.1,20.9)$ \\
$81.3 \pm 1.4$ \\
$2.8(2.1,4.1)$ \\
\hline
\end{tabular}

\begin{tabular}{c}
\hline Normal-weight controls $(n=20)$ \\
\hline $26.5(24,32)$ \\
$61.2 \pm 1.8$ \\
$22.6 \pm 0.3$ \\
$102.4 \pm 1.3$ \\
- \\
- \\
$164.1 \pm 9.8$ \\
$34.5(23.4,54.3)$ \\
$8.6(5.4,16.1)$ \\
$220 \pm 11$ \\
$54.5(30.1,96.6)$ \\
$7.71(6.76,8.27)$ \\
$12.1(10.1,15.2)$ \\
$87.4 \pm 1.4$ \\
$6.2(4.5,7.6)$ \\
\end{tabular}

\begin{tabular}{c}
\hline P-Value \\
\hline 0.99 \\
$<0.0001$ \\
$<0.0001$ \\
$<0.0001$ \\
- \\
- \\
$<0.0001$ \\
$<0.02$ \\
$<0.0001$ \\
0.004 \\
$<0.05$ \\
$<0.04$ \\
0.05 \\
0.004 \\
0.0001
\end{tabular}

short-term fast (15) and treatment with recombinant human IGF-1 during acute starvation or in chronically undernourished individuals with anorexia nervosa results in higher bone formation marker levels compared with placebo-treated individuals $(15,16,17)$. In anorexia nervosa, both leptin and IGF-1 are also positively correlated with $\operatorname{BMD}(18,19,20)$. At baseline, serum leptin $(P<0.0001)$ and IGF-1 levels $(P=0.004)$ were significantly lower in women with anorexia nervosa as compared to normal-weight controls. Additional hormones, which have been associated with adiposity and/or mineral metabolism, were measured including adiponectin, PYY, cortisol, insulin and glucose. Adiponectin and PYY levels were significantly higher whereas insulin and glucose levels were significantly lower in women with anorexia nervosa as compared to normal-weight controls (Table 1).

Baseline BMD and MAT data are reported in Table 2. BMD at the spine and hip were significantly lower in women with anorexia nervosa as compared to the normal-weight controls $(P \leq 0.0002$ for all). MAT at the L4 vertebra $(P<0.0001)$ and femoral metaphysis $(P=0.001)$ were significantly higher in the women with anorexia nervosa as compared to controls. Body weight and BMI were both inversely associated with MAT at the L4 vertebra (body weight: rho=-0.47, $P=0.001$; BMI: rho $=-0.51, P=0.0005)$ and MAT at the femoral metaphysis (body weight: rho $=-0.39, P=0.009$; BMI: rho $=-0.45, P<0.003$ ) (Fig. 1).

\section{Baseline associations between MAT, body composition and hormonal parameters}

At baseline, there were significant inverse associations between MAT of the spine and hip and spine and hip BMD (Table 3). MAT of both the spine and hip was also inversely correlated with subcutaneous adipose tissue (Table 3). In contrast, there were no associations between MAT and the visceral adipose tissue depot (Table 3). Leptin and

Table 2 Baseline bone mineral density and marrow adipose tissue of study subjects. We report (mean \pm S.E.M.) or median (interquartile range) when data were not normally distributed.

\begin{tabular}{|c|c|c|c|}
\hline & Anorexia nervosa $(n=26)$ & Normal-weight controls $(n=20)$ & P-Value \\
\hline \multicolumn{4}{|l|}{ Bone mineral density } \\
\hline Lumbar spine (L1-L4) (g/cm²) & $0.79(0.69,0.90)$ & $0.95(0.89,1.04)$ & 0.0002 \\
\hline Lateral spine (L2-L4) $\left(\mathrm{g} / \mathrm{cm}^{2}\right)$ & $0.59(0.53,0.64)$ & $0.76(0.72,0.83)$ & $<0.0001$ \\
\hline Total hip $\left(\mathrm{g} / \mathrm{cm}^{2}\right)$ & $0.77 \pm 0.03$ & $0.96 \pm 0.03$ & $<0.0001$ \\
\hline Femoral neck $\left(\mathrm{g} / \mathrm{cm}^{2}\right)$ & $0.67(0.58,0.73)$ & $0.83(0 . \overline{7} 6,0.96)$ & $<0.0001$ \\
\hline \multicolumn{4}{|l|}{ Marrow adipose tissue } \\
\hline L4 vertebra (lipid/water) & $0.98 \pm 0.10$ & $0.48 \pm 0.03$ & $<0.0001$ \\
\hline Femoral metaphysis (lipid/water) & $5.63(3.66,8.14)$ & $2.50(2.05,4.48)$ & 0.001 \\
\hline Femoral diaphysis (lipid/water) & $6.50 \pm 0.76$ & $5.58 \pm 0.62$ & NS \\
\hline Femoral epiphysis (lipid/water) & $7.47 \pm 0.58$ & $7.44 \pm 0.52$ & NS \\
\hline
\end{tabular}



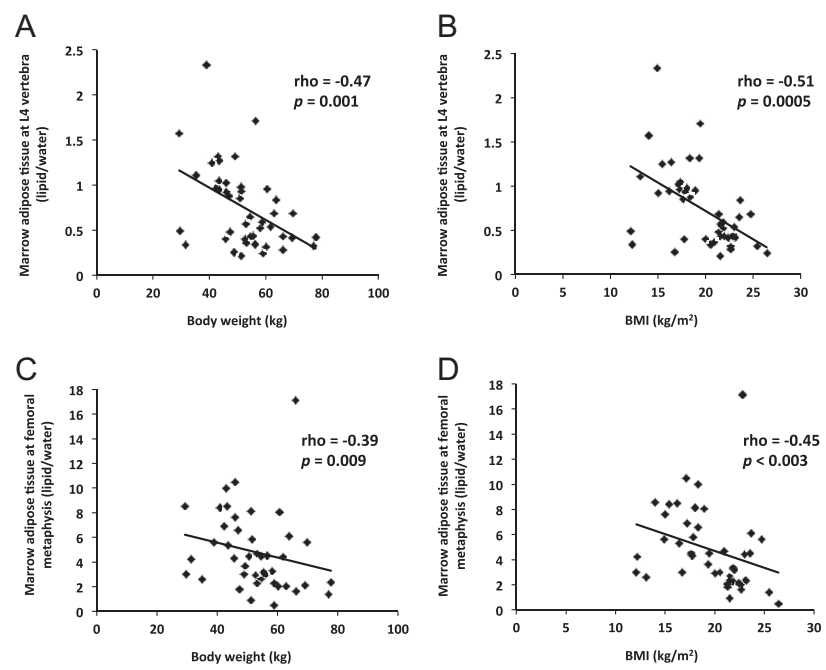

\section{Figure 1}

Both body weight and BMI were inversely associated with marrow adipose tissue (MAT) at the L4 vertebra and femoral metaphysis. Association between body weight and MAT at L4 vertebra (rho $=-0.47, P=0.001)($ panel $A)$; association between $\mathrm{BMI}$ and MAT at L4 vertebra (rho $=-0.51, P=0.0005)($ panel $B)$; association between body weight and MAT at the femoral metaphysis ( $r$ o $=-0.39, P=0.009)$ (panel $C$ ); association between BMI and MAT at the femoral metaphysis ( $r$ o $=-0.45$, $P<0.003$ ) (panel D). When the subject with the greatest $L 4$ MAT (2.4 lipid/water) was excluded, the associations remained significant (panel A: rho $=-0.44, P=0.003$ and panel $B$ : rho $=-0.48, P=0.001)$. When the subject with the greatest MAT at the femoral metaphysis (17.1 lipid/water) was excluded, the associations remained significant (panel C: rho $=-0.47, P<0.002$ and panel $\mathrm{D}$ : rho $=-0.52, P=0.0004)$.

HOMA-IR, a measure of insulin resistance, were inversely associated with MAT at baseline (Table 3), whereas for IGF1 , there was a trend toward an inverse association between IGF- 1 and MAT at the L4 vertebra (rho $=-0.31, P=0.05)$. In contrast, adiponectin was positively associated with MAT at the L4 vertebra (Table 3), consistent with murine data demonstrating that adiponectin is a hormone secreted by MAT (21).

There were significant positive associations between BMD at the spine and hip and the subcutaneous adipose tissue depot at baseline. Subcutaneous adipose tissue was significantly correlated with $\mathrm{BMD}$ at the lumbar (rho $=0.61, P<0.0001)$ and lateral (rho=0.64, $P<0.0001)$ spine, as well as BMD at the total hip (rho=0.61, $P<0.0001)$ and femoral neck (rho=0.58, $P<0.0001)$. There were no significant associations between BMD and visceral adipose tissue stores.
Similarly, IGF-1, insulin and leptin levels were all positively associated with BMD parameters. IGF-1 was associated with BMD of the total hip $(R=0.39, P=0.01)$ and femoral neck $(R=0.46, P<0.003)$ and insulin was positively associated with BMD of the lumbar ( $\mathrm{rho}=0.31$, $P<0.05)$ and lateral (rho $=0.40, P=0.008)$ spine, total hip $(\mathrm{rho}=0.38, P=0.01)$ and femoral neck (rho=0.41, $P=0.006)$. Leptin was positively associated with BMD of the lumbar (rho=0.47, $P<0.002)$ and lateral (rho=0.56, $P=0.0001)$ spine, total hip $($ rho $=0.50, \quad P<0.001)$ and femoral neck (rho=0.50, $P<0.001)$. In contrast, adiponectin and cortisol were inversely associated with all spine and hip BMD parameters (rho $=-0.30$ to -0.41 , $P<0.05$ for all associations), and there was a trend toward an inverse association between PYY and lumbar spine BMD (rho $=-0.30, P=0.06$ ).

\section{Changes in weight over 12 months}

After 12 months, a total of 28 subjects gained a median of $5.9 \%(2.4 \%, 14.7 \%)$ of their baseline weight. Seventeen subjects with anorexia nervosa (median weight gain: $8.6 \%$ (3.5\%, 18.6\%)) and 11 normal-weight controls (median weight gain: $3.2 \%(0.9 \%, 10.2 \%))$ gained weight during the study period. Subjects who lost weight during the 12 -month follow-up period lost a median of $2.1 \%(-6.3 \%$, $-1.2 \%$ ) of their baseline weight. The nine subjects with anorexia nervosa who lost weight during follow-up lost a median of $2.1 \%(-4.9 \%,-0.6 \%)$.

\section{Associations between weight gain and changes in body composition and hormonal parameters}

$B M D$

In subjects who gained weight, lumbar spine BMD increased a median of $1.3 \%(-0.5 \%, 3.0 \%)(P=0.03)$ and lateral spine BMD increased a median of $2.6 \%(0.2 \%$, $5.4 \%)(P<0.02)$. There were no significant changes in total hip or femoral neck BMD with weight gain. The subset of subjects with anorexia nervosa who gained weight over the 12-month study period also had significant increases in both lumbar spine $(2.4 \%(0.3 \%, 3.7 \%), P=0.01)$ and lateral spine $(3.9 \%(1.7 \%, 6.2 \%), P<0.005) \mathrm{BMD}$, whereas there were no significant changes in BMD in the subset of normal-weight controls who gained weight (Table 4).

\section{Subcutaneous and visceral adipose tissue}

Subcutaneous and visceral adipose tissue increased significantly in individuals who gained weight over the 
Table 3 Statistically significant $(P<0.05)$ baseline univariate associations between body composition parameters, hormonal parameters and marrow adipose tissue (MAT*).

\begin{tabular}{|c|c|c|c|c|}
\hline & \multicolumn{2}{|c|}{ MAT L4 vertebra (lipid/water) } & \multicolumn{2}{|c|}{ MAT femoral metaphysis (lipid/water) } \\
\hline & rho & $P$ & rho & $P$ \\
\hline BMD at lumbar spine $\left(\mathrm{g} / \mathrm{cm}^{2}\right)$ & -0.62 & $<0.0001$ & -0.47 & $<0.002$ \\
\hline BMD at lateral spine $(\mathrm{L} 2-\mathrm{L} 4)\left(\mathrm{g} / \mathrm{cm}^{2}\right)$ & -0.65 & $<0.0001$ & -0.62 & $<0.0001$ \\
\hline BMD at total hip $\left(\mathrm{g} / \mathrm{cm}^{2}\right)$ & -0.61 & $<0.0001$ & -0.50 & 0.0007 \\
\hline BMD at femoral neck (g/cm²) & -0.65 & $<0.0001$ & -0.49 & 0.001 \\
\hline Subcutaneous adipose tissue $\left(\mathrm{cm}^{2}\right)$ & -0.47 & 0.001 & -0.40 & 0.008 \\
\hline Visceral adipose tissue $\left(\mathrm{cm}^{2}\right)$ & - & - & - & - \\
\hline Leptin $(\mathrm{ng} / \mathrm{mL})$ & -0.45 & 0.003 & -0.35 & $<0.03$ \\
\hline IGF-1 (ng/mL) & -0.31 & 0.05 & - & - \\
\hline HOMA-IR & -0.41 & $<0.01$ & - & - \\
\hline Insulin $(\mu \mathrm{U} / \mathrm{mL})$ & -0.40 & $<0.01$ & & \\
\hline Glucose (mg/dL) & -0.35 & $<0.03$ & & \\
\hline Adiponectin $(\mu \mathrm{g} / \mathrm{mL})$ & 0.38 & 0.01 & - & - \\
\hline PYY (pg/mL) & - & - & - & - \\
\hline Cortisol ( $\mu g / d L)$ & - & - & - & - \\
\hline
\end{tabular}

*There were no associations between the parameters and MAT at the femoral diaphysis or MAT at the femoral epiphysis.

12-month follow-up. Compared to baseline, subcutaneous adipose tissue increased a median of $35.6 \%(9.2 \%, 77.3 \%)$ $(P<0.0001)$ and visceral adipose tissue increased a median of $38.8 \%(-13.4 \%, 101.6 \%) \quad(P=0.03)$. In the subset of subjects with anorexia nervosa who gained weight over the study period $(n=17)$, subcutaneous adipose tissue increased a median of $67.3 \%(26.0 \%, 158.8 \%)$ $(P=0.0002)$, whereas visceral adipose tissue did not increase significantly (Table 4). In normal-weight subjects, subcutaneous adipose tissue also increased significantly $(P=0.002)$, and there was a trend toward an increase in visceral adipose tissue $(P=0.05)$ in those who gained weight during the 12-month follow-up.

\section{Marrow adipose tissue}

MAT increased significantly in the epiphysis in individuals who gained weight over 12 months. In subjects who gained weight, epiphyseal MAT increased a median of $16.3 \%(-15.3 \%, 56.5 \%)(P<0.03)$ (Table 4$)$. In the subset of subjects with anorexia nervosa who gained weight during the study $(n=17)$, epiphyseal MAT also increased significantly $(25.3 \%(12.2 \%, 99.7 \%), P=0.006)$. Of the 17 women with anorexia nervosa who gained weight during the study, 53\% $(n=9)$ achieved a normal weight, defined as $\mathrm{BMI} \geq 18.5 \mathrm{~kg} / \mathrm{m}^{2}$ and $\%$ ideal body weight of $\geq 85 \%$. There were no significant differences in \% change in spine

Table 4 Changes in body composition and hormonal parameters after 12-months in the subset of subjects who gained weight during the study $(n=28)$. Data are reported as median (interquartile range).

\begin{tabular}{|c|c|c|c|c|c|c|}
\hline & \multicolumn{6}{|c|}{ Median \% change in subjects who gained weight during the 12 -month study } \\
\hline & All $(n=28)$ & $P$-Value & Anorexia nervosa $(n=17)$ & $P$-Value & Normal-weight $(n=11)$ & $P$-Value \\
\hline \multicolumn{7}{|l|}{ Bone mineral density } \\
\hline Lumbar spine (L1-L4) & $1.3(-0.5,3.0)$ & 0.03 & $2.4(0.3,3.7)$ & 0.01 & $-0.1(-1.5,1.3)$ & 0.93 \\
\hline Lateral spine (L2-L4) & $2.6(0.2,5.4)$ & $<0.02$ & $3.9(1.7,6.2)$ & $<0.005$ & $0.1(-2.5,1.4)$ & 0.51 \\
\hline Total hip & $-1.3(-3.8,1.1)$ & 0.14 & $-0.8(-3.7,1.1)$ & 0.23 & $-1.3(-4.5,1.8)$ & 0.30 \\
\hline Femoral neck & $0.2(-3.1,2.8)$ & 0.61 & $-0.3(-3.4,2.9)$ & 0.37 & $0.7(-2.1,2.8)$ & 0.74 \\
\hline \multicolumn{7}{|l|}{ Marrow adipose tissue } \\
\hline L4 vertebra & $-0.6(-8.2,28.4)$ & 0.57 & $6.3(-25.5,76.8)$ & 0.50 & $-1.1(-6.1,6.3)$ & 0.46 \\
\hline Femoral metaphysis & $1.9(-27.7,40.3)$ & 0.70 & $14.3(-28.2,31.8)$ & 0.26 & $-15.9(-31.1,49.1)$ & 0.92 \\
\hline Femoral diaphysis & $1.1(-15.5,51.8)$ & 0.15 & $7.0(-11.3,111.0)$ & 0.09 & $-1.0(-31.9,51.8)$ & 0.99 \\
\hline Femoral epiphysis & $16.3(-15.3,56.5)$ & $<0.03$ & $25.3(12.2,99.7)$ & 0.006 & $4.0(-36.4,25.9)$ & 0.92 \\
\hline Subcutaneous adipose tissue & $35.6(9.2,77.3)$ & $<0.0001$ & $67.3(26.0,158.8)$ & 0.0002 & $9.7(6.0,58.8)$ & 0.002 \\
\hline Visceral adipose tissue & $38.8(-13.4,101.6)$ & 0.03 & $4.4(-15.8,113.3)$ & 0.22 & $44.6(-0.8,105.1)$ & 0.05 \\
\hline Leptin & $114.5(5.6,183.4)$ & 0.0003 & $147.6(36.7,245.6)$ & 0.001 & $13.0(-1.2,151.1)$ & 0.08 \\
\hline IGF-1 & $-0.8(-15.0,12.0)$ & 0.88 & $2.2(-14.8,11.9)$ & 0.71 & $-5.3(-15.6,14.8)$ & 0.85 \\
\hline PYY & $24.2(-8.9,51.4)$ & 0.13 & $19.8(-28.1,34.8)$ & 0.90 & $40.5(12.2,154.7)$ & $<0.04$ \\
\hline Adiponectin & $-7.9(-17.9,18.7)$ & 0.30 & $-13.6(-33.9,18.3)$ & 0.21 & $-0.2(-10.3,19.6)$ & 0.48 \\
\hline HOMA-IR & $32.0(-3.7,97.4)$ & $<0.004$ & $61.4(6.8,97.5)$ & $<0.002$ & $-0.9(-17.3,57.9)$ & 0.26 \\
\hline
\end{tabular}

https://eje.bioscientifica.com 
or femoral MAT when comparing women who achieved a normal weight to those who gained weight but did not achieve a normal weight $(P=0.27-0.96)$. There were also no significant changes in MAT in normal-weight subjects who gained weight during the study.

Of the 26 women with anorexia nervosa, $58 \%(n=15)$ reported having at least one menstrual bleed during the 12-month study. When comparing \% change in epiphyseal MAT in women with anorexia nervosa who gained weight and had at least one menstrual bleed (median change: $21.6 \%(-11.8 \%, 60.9 \%))$ to $\%$ change in epiphyseal MAT in women who gained weight but were amenorrheic (median change: $35.7 \%$ (14.3\%, 154.9\%)), \% change in MAT was lower in women who had a menstrual bleed but the difference was not statistically significant $(P=0.23)$.

In subjects who lost weight over the 12-month follow-up, there was a decrease in epiphyseal MAT (median change: $-14.4 \%(-35.5 \%,-0.8 \%) ; P<0.02)$. There was a significant, positive association between $\%$ change in weight and \% change in epiphyseal MAT over the 12-month study (rho $=0.51, P<0.001$ ) (Fig. 2). When we excluded the two subjects who had the greatest increase in weight over the course of the study (weight gain: 55.1\% and $39.4 \%$ ), the association between \% change in weight

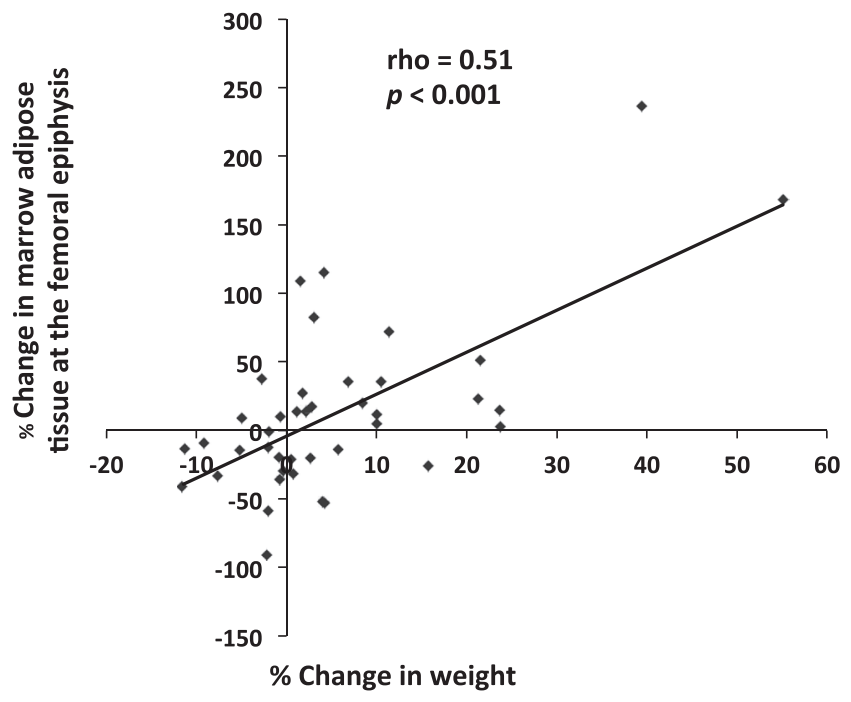

\section{Figure 2}

There was a significant positive association between \% change in weight and \% change in marrow adipose tissue at the femoral epiphysis ( $r$ o $=0.51, P<0.001$ ) over the 12-month study. When we excluded the two subjects who gained more weight (weight gain: $55.1 \%$ and 39.4\%) than the rest of the subjects, the association remained significant ( $r$ o $=0.44$, $P<0.01)$. and \% change in epiphyseal MAT remained significant (rho=0.44, $P<0.01$ ). There was no significant change in vertebral MAT in subjects who gained ( $P=0.57$; Table 4$)$ or lost $(P=0.27)$ weight over the follow-up period.

\section{Hormonal parameters}

In subjects who gained weight and in those who lost weight, leptin levels changed significantly during the study. Leptin levels rose a median of $114.5 \%(5.6 \%, 183.4 \%)(P=0.0003)$ over the 12-month study in subjects who gained weight (Table 4). In subjects who lost weight, leptin levels changed by a median of $-36.2 \%(-59.6 \%, 4.9 \%)(P=0.01)$. IGF-1 levels did not change significantly in subjects who gained weight $(P=0.88)$ but dropped $12 \pm 4.9 \%(P=0.03)$ in subjects who lost weight. HOMA-IR increased significantly in the subset of subjects with anorexia nervosa $(P<0.002)$ who gained weight during the study but did not change significantly in the subset of normal-weight subjects who gained weight (Table 4). In contrast, PYY levels did not change significantly in the subset of women with anorexia nervosa who gained weight but did increase significantly in the subset of normal-weight controls who gained weight during the 12 -month study (median change: $40.5 \%$ $(12.2 \%, 154.7 \%), P<0.04)$.

\section{Associations between changes in MAT and changes in body composition and hormonal parameters}

\section{MAT and BMD}

In contrast to the relationship between MAT and BMD observed at baseline, \% change in epiphyseal MAT was significantly and positively associated with \% change in lateral spine BMD (rho=0.35, $P<0.03$ ) (Fig. 3). No associations were observed between changes in MAT and changes in hip BMD over the course of the study.

\section{MAT and subcutaneous and visceral adipose tissue}

Similarly, \% change in MAT at the femoral epiphysis was positively associated with \% change in subcutaneous adipose tissue over the course of the study $(\mathrm{rho}=0.48$, $P=0.002$ ) (Fig. 4). When we excluded the two subjects who had the greatest increase in subcutaneous adipose tissue $(790 \%$ and $415 \%)$ during the study, the association remained significant (rho $=0.39, P<0.02$ ). There were no significant correlations between \% change in MAT and \% change in the visceral adipose tissue depot over the course of the 12-month study. 


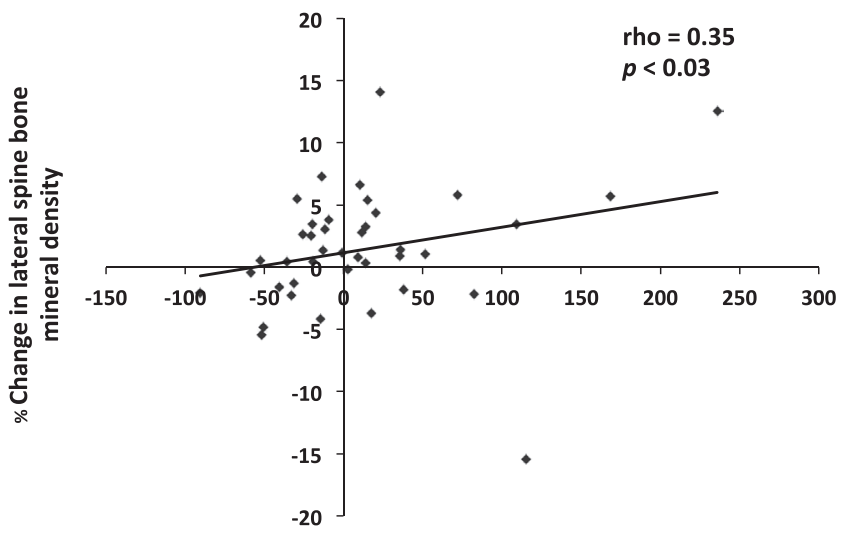

$\%$ Change in marrow adipose tissue at the femoral epiphysis

\section{Figure 3}

$\%$ change in marrow adipose tissue at the femoral epiphysis was significantly and positively associated with $\%$ change in lateral spine bone mineral density (rho $=0.35, P<0.03$ ).

\section{MAT and hormonal parameters}

There were significant positive associations between $\%$ change in leptin and \% change in MAT after 12 months, opposite to the observed baseline association. \% change in leptin was positively associated with \% change in MAT at the $\mathrm{L} 4$ vertebra $(\mathrm{rho}=0.36, P=0.02)$ and $\%$ change in MAT at the epiphysis (rho $=0.37, P=0.02$ ). Similarly, opposite to

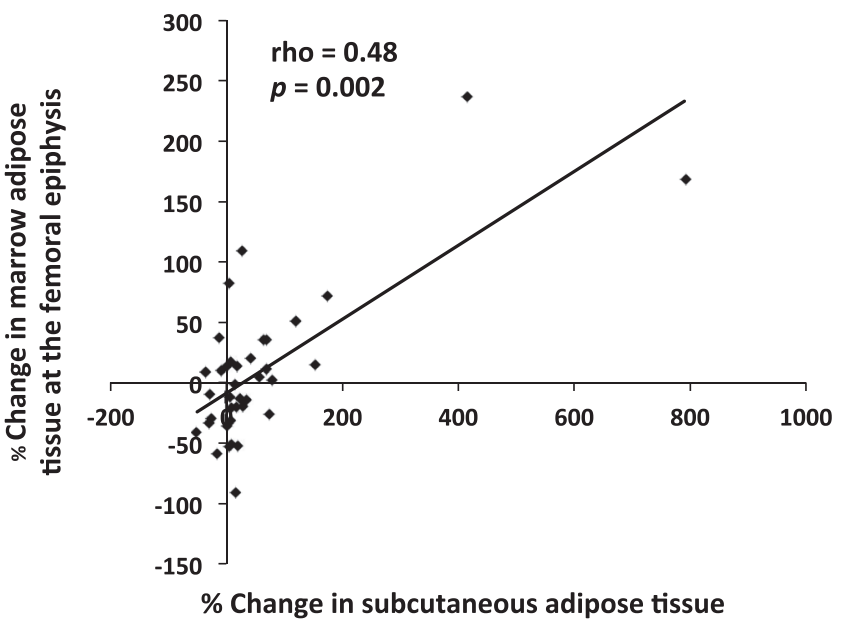

\section{Figure 4}

$\%$ change in marrow adipose tissue at the femoral epiphysis was significantly and positively associated with $\%$ change in subcutaneous adipose tissue ( $r$ o $=0.48, P=0.002$ ). When we excluded the two subjects who had higher gains in subcutaneous adipose tissue (790\% and $415 \%)$ than the rest of the subjects, the association remained significant ( $r h o=0.39$, $P<0.02)$. the observed baseline associations, there were significant positive associations between \% change in MAT and \% change in insulin and HOMA-IR. \% change in insulin and $\%$ change in HOMA-IR were significantly correlated with $\%$ change in MAT at the L4 vertebra (insulin: rho $=0.40$, $P=0.01$ and HOMA-IR: rho $=0.39, P=0.01)$ and MAT at the femoral diaphysis (insulin: $r h o=0.36, P<0.03$ and HOMA-IR: rho $=0.32, P<0.05$ ) and epiphysis (insulin: rho=0.41, $P=0.01$ and HOMA-IR: rho=0.40, $P<0.02)$. There were no significant associations between change in IGF-1, PYY, adiponectin, cortisol or glucose levels and change in MAT.

\section{Discussion}

We have shown that in a population of lean women, the association between MAT and body weight is distinctly different in the steady state as compared to periods of subacute change. In the steady state, body weight is inversely associated with MAT such that individuals with anorexia nervosa, a model of chronic undernutrition, have significantly higher levels of MAT compared to normalweight women. In sharp contrast, with longitudinal changes in weight, we demonstrate that MAT levels increase with weight gain and decrease with weight loss over a 12-month period.

In states of chronic undernutrition such as anorexia nervosa, subcutaneous and visceral adipose tissue stores are low compared to those of normal-weight individuals $(1,2)$. In contrast, MAT stores, which are located in the bone marrow microenvironment, are elevated in women with anorexia nervosa $(2,22)$. We have previously shown that women with a history of anorexia nervosa who have recovered their weight and menses have less MAT as compared to women with active anorexia nervosa, and in fact, their levels of MAT are comparable to those of normalweight individuals (10). Therefore, we hypothesized that subacute weight gain would result in loss of MAT. Instead, we demonstrate the opposite finding - MAT, similar to the subcutaneous and visceral adipose tissue depots, increases with weight gain and levels decrease with weight loss. Therefore, with short-term changes, MAT follows the same pattern as the subcutaneous and visceral adipose tissue depots. These findings suggest that MAT is a dynamic depot that acts differentially in the steady state as compared to periods of recent weight change.

The visceral adipose tissue and intermuscular adipose tissue depots, another ectopic fat depot which has been associated with insulin resistance (23), follow a similar 
pattern of dynamic change with short-term vs longterm weight gain in women with anorexia nervosa. With short-term weight recovery (4-18 weeks between lowweight and BMI normalization), women with anorexia nervosa have significantly more visceral adipose tissue and intermuscular adipose tissue as compared to normalweight control subjects with similar BMI $(1,24)$. Yet, with longer-term weight recovery (weight maintenance for approximately 1 year), women with anorexia nervosa have similar amounts of visceral and intermuscular adipose tissue compared to control subjects (24). These similarities in longitudinal changes in marrow, visceral and intermuscular adipose tissue may provide insight into the metabolic functions of MAT, which presently remain unknown.

Some insight into the metabolic function of MAT has been provided by studies in overweight and obese individuals. In contrast to our findings in lean women, in overweight and obese women, MAT is positively associated with VAT (25), which may suggest that MAT may serve a different function in states of nutritional excess as compared to nutritional insufficiency. In overweight women with type 2 diabetes mellitus, individuals with hemoglobin A1C levels $>7 \%$ have higher levels of MAT compared with individuals with hemoglobin A1C levels $\leq 7 \%$ (26), suggesting an association between MAT and glucose metabolism in individuals with diabetes mellitus. Similarly, in a population of morbidly obese women undergoing Roux-en-Y gastric bypass surgery for weight loss in whom vertebral MAT was measured before and 6 months after surgery, there was no change in MAT observed in the non-diabetic women who lost a mean of $25 \mathrm{~kg}$ ( $21 \%$ of baseline weight) during the 6-month follow-up (27). In contrast, in the diabetic women who lost a similar amount of weight (mean of $27.8 \mathrm{~kg}$ or $26.2 \%$ of baseline weight), MAT decreased a mean of $7.5 \%$ (27). Although there was no association between the drop in MAT and normalization of hemoglobin A1C levels in the women with diabetes mellitus in this study, these findings also suggest a potential association between glucose homeostasis and MAT (27). Our finding of a positive association between change in MAT and change in HOMA-IR also suggests an association between MAT and glucose metabolism, but in contrast to prior studies, all of our study participants were lean and non-diabetic.

Importantly, changes in MAT in the population of women with diabetes mellitus who underwent Roux-en Y gastric bypass surgery were also directionally concordant with the changes observed in our study subjects - MAT levels decreased with weight loss (27). This has also been observed in obese individuals undergoing sleeve gastrectomy, another surgical weight-loss procedure (28). In a 12-month longitudinal study, change in weight was positively associated with change in MAT (28) in postsleeve gastrectomy patients, again congruent with our findings in lean women.

Our data also demonstrate a significant positive association between change in MAT and change in BMD over the 12-month study, in contrast to the inverse association between MAT and BMD observed in the steady state. Whereas MAT and BMD are inversely associated in a majority of populations, including healthy men and women $(29,30,31,32)$, there are disease states, such as HIV, in which BMD and MAT levels are both reduced (33). Similarly, immediately after birth, MAT begins to accumulate in humans and increases in MAT during growth are coincident with increases in bone accrual (34). As spine BMD also increased with weight gain during our 12-month study, our findings are consistent with the relationship observed between MAT and BMD during periods of bone accrual - increases in both. Importantly, we only observed significant changes in MAT at the femoral epiphysis over the 12-month study. The reason for this is not known but the epiphysis contains more MAT than any of the other sites measured (Table 2). Therefore, we can speculate that perhaps changes in MAT are observed at this site earlier than at other sites or that the changes at this site are more clearly observed, given its high fat content.

In conclusion, we demonstrate significant differences when comparing steady-state associations between weight, BMD and MAT and associations during periods of subacute changes in weight; although at baseline MAT levels are inversely associated with BMI and weight, with weight gain, levels of MAT also increase. Our data provide further evidence that MAT is a dynamic depot and may serve different functions in states of nutrient sufficiency versus nutrient insufficiency. Gaining greater insight into these differences will be critical to understanding the role and function of MAT in humans.

\section{Declaration of interest}

The authors declare that there is no conflict of interest that could be perceived as prejudicing the impartiality of this study.

\section{Funding}

The project described was supported by Harvard Catalyst/The Harvard Clinical and Translational Science Center (National Center for Advancing Translational Sciences, National Institutes of Health Award UL 1TR002541) and NIH grants R24 DK084970 and P30 DK040561. The content is solely the 
responsibility of the authors and does not necessarily represent the official views of the National Institutes of Health.

\section{Acknowledgements}

The authors thank the nurses and bionutritionists of the MGH Translational and Clinical Research Center for their expert care.

\section{References}

1 Mayer L, Walsh BT, Pierson RN Jr, Heymsfield SB, Gallagher D, Wang J, Parides MK, Leibel RL, Warren MP, Killory E et al. Body fat redistribution after weight gain in women with anorexia nervosa. American Journal of Clinical Nutrition 200581 1286-1291. (https:// doi.org/10.1093/ajcn/81.6.1286)

2 Bredella MA, Fazeli PK, Miller KK, Misra M, Torriani M, Thomas BJ, Ghomi RH, Rosen CJ \& Klibanski A. Increased bone marrow fat in anorexia nervosa. Journal of Clinical Endocrinology and Metabolism 200994 2129-2136. (https://doi.org/10.1210/jc.2008-2532)

3 Grinspoon S, Thomas E, Pitts S, Gross E, Mickley D, Miller K, Herzog D \& Klibanski A. Prevalence and predictive factors for regional osteopenia in women with anorexia nervosa. Annals of Internal Medicine 2000133 790-794. (https://doi.org/10.7326/00034819-133-10-200011210-00011)

4 Rigotti NA, Neer RM, Skates SJ, Herzog DB \& Nussbaum SR. The clinical course of osteoporosis in anorexia nervosa. A longitudinal study of cortical bone mass. JAMA 1991265 1133-1138. (https://doi. org/10.1001/jama.1991.03460090081037)

5 Miller KK, Grinspoon SK, Ciampa J, Hier J, Herzog D \& Klibanski A. Medical findings in outpatients with anorexia nervosa. Archives of Internal Medicine 2005165 561-566. (https://doi.org/10.1001/ archinte.165.5.561)

6 Faje AT, Fazeli PK, Miller KK, Katzman DK, Ebrahimi S, Lee H, Mendes N, Snelgrove D, Meenaghan E, Misra M et al. Fracture risk and areal bone mineral density in adolescent females with anorexia nervosa. International Journal of Eating Disorders 201447 458-466. (https://doi.org/10.1002/eat.22248)

7 Fazeli PK \& Klibanski A. Effects of anorexia nervosa on bone metabolism. Endocrine Reviews 201839 895-910. (https://doi. org/10.1210/er.2018-00063)

8 Singhal V, Tulsiani S, Campoverde KJ, Mitchell DM, Slattery M, Schorr M, Miller KK, Bredella MA, Misra M \& Klibanski A. Impaired bone strength estimates at the distal tibia and its determinants in adolescents with anorexia nervosa. Bone 2018106 61-68. (https:// doi.org/10.1016/j.bone.2017.07.009)

9 Schellinger D, Lin CS, Hatipoglu HG \& Fertikh D. Potential value of vertebral proton MR spectroscopy in determining bone weakness. American Journal of Neuroradiology 200122 1620-1627.

10 Fazeli PK, Bredella MA, Freedman L, Thomas BJ, Breggia A, Meenaghan E, Rosen CJ \& Klibanski A. Marrow fat and preadipocyte factor-1 levels decrease with recovery in women with anorexia nervosa. Journal of Bone and Mineral Research 201227 1864-1871. (https://doi.org/10.1002/jbmr.1640)

11 American Psychiatric Association. Diagnostic and Statistical Manual of Mental Disorders (DSM-IV), 4th ed. American psychiatric Association: Washington, DC, 1994.

12 Johnson J \& Dawson-Hughes B. Precision and stability of dual-energy $\mathrm{X}$-ray absorptiometry measurements. Calcified Tissue International 199149 174-178. (https://doi.org/10.1007/BF02556113)

13 Fazeli PK, Faje AT, Cross EJ, Lee H, Rosen CJ, Bouxsein ML \& Klibanski A. Serum FGF-21 levels are associated with worsened radial trabecular bone microarchitecture and decreased radial bone strength in women with anorexia nervosa. Bone 201577 6-11. (https://doi. org/10.1016/j.bone.2015.04.001)
14 Welt CK, Chan JL, Bullen J, Murphy R, Smith P, DePaoli AM, Karalis A \& Mantzoros CS. Recombinant human leptin in women with hypothalamic amenorrhea. New England Journal of Medicine 2004351 987-997. (https://doi.org/10.1056/NEJMoa040388)

15 Grinspoon SK, Baum HB, Peterson S \& Klibanski A. Effects of rhIGF-I administration on bone turnover during short-term fasting. Journal of Clinical Investigation 199596 900-906. (https://doi.org/10.1172/ JCI118137)

16 Grinspoon S, Baum H, Lee K, Anderson E, Herzog D \& Klibanski A. Effects of short-term recombinant human insulin-like growth factor I administration on bone turnover in osteopenic women with anorexia nervosa. Journal of Clinical Endocrinology and Metabolism 199681 3864-3870. (https://doi.org/10.1210/ jcem.81.11.8923830)

17 Misra M, McGrane J, Miller KK, Goldstein MA, Ebrahimi S, Weigel T \& Klibanski A. Effects of rhIGF-1 administration on surrogate markers of bone turnover in adolescents with anorexia nervosa. Bone 200945 493-498. (https://doi.org/10.1016/j.bone.2009.06.002)

18 Fazeli PK, Bredella MA, Misra M, Meenaghan E, Rosen CJ, Clemmons DR, Breggia A, Miller KK \& Klibanski A. Preadipocyte factor- 1 is associated with marrow adiposity and bone mineral density in women with anorexia nervosa. Journal of Clinical Endocrinology and Metabolism 201095 407-413. (https://doi. org/10.1210/jc.2009-1152)

19 Legroux-Gerot I, Vignau J, Biver E, Pigny P, Collier F, Marchandise X, Duquesnoy B \& Cortet B. Anorexia nervosa, osteoporosis and circulating leptin: the missing link. Osteoporosis International 201021 1715-1722. (https://doi.org/10.1007/s00198-009-1120-x)

20 Grinspoon S, Miller K, Coyle C, Krempin J, Armstrong C, Pitts S, Herzog D \& Klibanski A. Severity of osteopenia in estrogen-deficient women with anorexia nervosa and hypothalamic amenorrhea. Journal of Clinical Endocrinology and Metabolism 199984 2049-2055. (https://doi.org/10.1210/jcem.84.6.5792)

21 Cawthorn WP, Scheller EL, Learman BS, Parlee SD, Simon BR, Mori H, Ning X, Bree AJ, Schell B, Broome DT et al. Bone marrow adipose tissue is an endocrine organ that contributes to increased circulating adiponectin during caloric restriction. Cell Metabolism 201420 368-375. (https://doi.org/10.1016/j.cmet.2014.06.003)

22 Ecklund K, Vajapeyam S, Feldman HA, Buzney CD, Mulkern RV, Kleinman PK, Rosen CJ \& Gordon CM. Bone marrow changes in adolescent girls with anorexia nervosa. Journal of Bone and Mineral Research 201025 298-304. (https://doi.org/10.1359/ jbmr.090805)

23 Goodpaster BH, Thaete FL \& Kelley DE. Thigh adipose tissue distribution is associated with insulin resistance in obesity and in type 2 diabetes mellitus. American Journal of Clinical Nutrition 2000 71 885-892. (https://doi.org/10.1093/ajcn/71.4.885)

24 Mayer LE, Klein DA, Black E, Attia E, Shen W, Mao X, Shungu DC, Punyanita M, Gallagher D, Wang J et al. Adipose tissue distribution after weight restoration and weight maintenance in women with anorexia nervosa. American Journal of Clinical Nutrition 200990 1132-1137. (https://doi.org/10.3945/ajcn.2009.27820)

25 Bredella MA, Torriani M, Ghomi RH, Thomas BJ, Brick DJ, Gerweck AV, Rosen CJ, Klibanski A \& Miller KK. Vertebral bone marrow fat is positively associated with visceral fat and inversely associated with IGF-1 in obese women. Obesity 201119 49-53. (https://doi.org/10.1038/oby.2010.106)

26 Baum T, Yap SP, Karampinos DC, Nardo L, Kuo D, Burghardt AJ, Masharani UB, Schwartz AV, Li X \& Link TM. Does vertebral bone marrow fat content correlate with abdominal adipose tissue, lumbar spine bone mineral density, and blood biomarkers in women with type 2 diabetes mellitus? Journal of Magnetic Resonance Imaging 2012 35 117-124. (https://doi.org/10.1002/jmri.22757)

27 Schafer AL, Li X, Schwartz AV, Tufts LS, Wheeler AL, Grunfeld C, Stewart L, Rogers SJ, Carter JT, Posselt AM et al. Changes in vertebral bone marrow fat and bone mass after gastric bypass surgery: a 
pilot study. Bone 201574 140-145. (https://doi.org/10.1016/j. bone.2015.01.010)

28 Bredella MA, Greenblatt LB, Eajazi A, Torriani M \& Yu EW. Effects of Roux-en-Y gastric bypass and sleeve gastrectomy on bone mineral density and marrow adipose tissue. Bone 201795 85-90. (https://doi. org/10.1016/j.bone.2016.11.014)

29 Shen W, Chen J, Punyanitya M, Shapses S, Heshka S \& Heymsfield SB. MRI-measured bone marrow adipose tissue is inversely related to DXA-measured bone mineral in Caucasian women. Osteoporosis International 200718 641-647. (https://doi.org/10.1007/s00198-0060285-9)

30 Di Iorgi N, Rosol M, Mittelman SD \& Gilsanz V. Reciprocal relation between marrow adiposity and the amount of bone in the axial and appendicular skeleton of young adults. Journal of Clinical Endocrinology and Metabolism 200893 2281-2286. (https://doi. org/10.1210/jc.2007-2691)
31 Wren TA, Chung SA, Dorey FJ, Bluml S, Adams GB \& Gilsanz V. Bone marrow fat is inversely related to cortical bone in young and old subjects. Journal of Clinical Endocrinology and Metabolism 201196 782-786. (https://doi.org/10.1210/jc.2010-1922)

32 Shen W, Scherzer R, Gantz M, Chen J, Punyanitya M, Lewis CE \& Grunfeld C. Relationship between MRI-measured bone marrow adipose tissue and hip and spine bone mineral density in AfricanAmerican and Caucasian participants: the CARDIA study. Journal of Clinical Endocrinology and Metabolism 201297 1337-1346. (https:// doi.org/10.1210/jc.2011-2605)

33 Huang JS, Mulkern RV \& Grinspoon S. Reduced intravertebral bone marrow fat in HIV-infected men. AIDS 200216 1265-1269. (https:// doi.org/10.1097/00002030-200206140-00009)

34 Kricun ME. Red-yellow marrow conversion: its effect on the location of some solitary bone lesions. Skeletal Radiology 198514 10-19. (https://doi.org/10.1007/BF00361188)

Received 9 October 2018

Revised version received 12 December 2018

Accepted 18 December 2018 